\title{
Evaluation of the somatosensory evoked blink response in patients with neurological disorders
}

\author{
Hideto Miwa, Yoshio Yamaji, Hirokazu Abe, Yoshikuni Mizuno
}

\begin{abstract}
Background-The somatosensory evoked blink response (SBR) is a characteristic reflex blink elicited by electrical stimulation of peripheral nerves or other anatomical sites.

Methods-139 patients with neurological disorders were examined for presence of the SBR. Although the SBR was not usually elicitable, it was present in a subset of patients with Parkinson's disease and with hemifacial spasm. It was also present in a patient with Guillain-Barré syndrome before the recovery phase. The latency of the EMG activities responsible for the SBR was significantly shorter than that of the startle blink.

Conclusions-The SBR is not a variant of the startle blink, but is a release phenomenon transmitted via the brainstem reticular formation. This response may be clinically relevant in disorders associated with brainstem lesions and abnormal blinking.
\end{abstract}

(F Neurol Neurosurg Psychiatry 1996;60:539-543)

Keywords: blink reflex; hemifacial spasm; Parkinson's disease

The somatosensory evoked blink response (SBR) is a newly reported blink response elicited by electrical stimulation of peripheral nerves or various other anatomical sites. ${ }^{1} \mathrm{We}$ previously reported that the SBR found in some patients with Miller Fisher syndrome disappeared during recovery ${ }^{1}$ and that a pronounced SBR was also seen in a patient with posthypoxic intention myoclonus (LanceAdams syndrome). ${ }^{2}$ Although it has been speculated that the SBR is a release phenomenon transmitted via the brainstem reticular formation, ${ }^{12}$ the actual pathophysiological mechanism still remains uncertain. However, the SBR may be a useful electrophysiological method for detecting some functional abnormalities of the CNS, because it can be easily

Received 10 Octobe
and in revised form

16 January 1996

Accepted 24 January 1996 recorded when present. In this study, we investigated potential clinical uses and the actual pathophysiological basis of the SBR by examining the SBR in various neurological conditions. Its relevance in disorders shown to have increased excitabilities of neurons responsible for blinking, such as Parkinson's disease, ${ }^{3}$ hemifacial spasm, ${ }^{4}$ and dystonia, ${ }^{5-7}$ is discussed.

\section{Patients and methods}

PATIENTS

All patients $(n=139)$ were outpatients or inpatients admitted to the hospital of Juntendo University School of Medicine. The clinical diagnosis was confirmed by board certified neurologists aided by brain CT or MRI, and routine laboratory tests. Verbal informed consent was obtained from all the participants.

\section{ELECTROMYOGRAPHICAL METHODS FOR} EVOKING THE SOMATOSENSORY EVOKED BLINK RESPONSE

Patients were requested to lie on a bed or sit in an armchair in a quiet room. A pair of surface electrodes were placed on the inferior portion of the orbicularis oculi muscles bilaterally. Electric shocks of $0.1 \mathrm{~ms}$ duration were applied to the cutaneous surface above the peripheral nerve trunk; for a routine examination, the median nerve was stimulated at the wrist above the supramaximal intensity. It has been shown that the SBR can be elicited by a stimulation at an intensity below the motor threshold; ${ }^{2}$ however, supramaximum intensity stimuli were given in this study, because the relation between the stimulus intensity and the appearance of the SBR has not been fully determined. With reference to properties of the SBR reported earlier, ${ }^{12}$ we made tentative criteria for the presence of the SBR for the present study (table 1). Latency and amplitude of the obtained SBRs were measured by inspection of superimposed responses. When the SBR could be evoked, repetitive stimulation was performed at a frequency higher than $0.1 \mathrm{~Hz}$-such as $1.0,0.5$, or $0.2 \mathrm{~Hz}$-for evaluating possible habituation. When a generalised startle jerk was elicited by median nerve stimulation, the surface electrodes were placed on several muscle groups such as the sternocleidomastoideus muscle, the biceps muscle, or the quadriceps muscle. EMG discharges of these muscles were recorded to distinguish the SBR from the startle blink. In all subjects, routine blink reflex studies were also performed. The supraorbital nerve was stimulated, and able 1 Criteria for the SBR elicited by electrical stimulation of the median nerve The latency $60 \mathrm{~ms}$

response is a bilaterat response (at times, its appearance can be slightly mor nounced on the side ipsilateral to the stimulation)

The EMG response is a phasic response with a duration of less than $100 \mathrm{~ms}$

If three consecutive electrical stimulations at intervals of at least 10 seconds fail to elicit any obvious EMG discharges, the SBR is considered negative

The amplitude of the obtained EMG response is at least threefold greater than the baseline activities of the orbicularis oculi muscles by inspection 


\begin{tabular}{lll}
\hline & $\begin{array}{l}\text { No of patients } \\
\text { studied }\end{array}$ & $\begin{array}{l}\text { No of SBR } \\
\text { positive patients }\end{array}$ \\
\hline $\begin{array}{l}\text { Disorders with increased excitabilities } \\
\text { of the blink reflex pathway: }\end{array}$ & \\
$\begin{array}{l}\text { Hemifacial spasm } \\
\text { Parkinson's disease }\end{array}$ & 31 & 9 \\
Dystonia & 16 & 5 \\
$\quad$ Meige's syndrome & 13 & 1 \\
$\quad$ Others & 18 & 1 \\
Other disorders: & 16 & 2 \\
Cerebrovascular disease & 12 & 2 \\
Multiple sclerosis & 12 & 1 \\
Spinocerebellar degeneration $\dagger$ & 13 & 2 \\
Peripheral nerve disorders & 3 & 0 \\
Exaggerated startle disorders & 5 & 0 \\
Others & &
\end{tabular}

^Nine patients with torticollis, five with writer's cramp, and four with generalised dystonia. tThree patients with progressive supranuclear palsy, three with olivopontocerebellar atrophy, three patients with progressive supranuclear palsy, three with olivopontocerebellar atrophy, three with late cerebellar cortical atrophy, one with corticobasal torubropallidoluysian atrophy, and one with Friedreich's ataxia. sarcoidosis.
Table 2 The somotaosensory evoked blink response in neurological disorders

but also to the cutaneous surface about $1 \mathrm{~cm}$ lateral to that point. In three of the SBR positive patients, the SBR could be elicited by stimulation of lower extremity peripheral nerves such as the sural nerve or the posterior tibial nerve. The latency of the SBR elicited by the stimulation of the lower limbs was longer that of the median nerve SBR (fig 1). In most of the SBR positive patients, the SBR was a delicate phenomenon. Habituation occurred easily, and the SBR soon disappeared after repeated stimulation. When the stimuli were repeated with interstimulus intervals of at least $10 \mathrm{~s}$, the SBR was reliably elicited; however, it occasionally became less evident, even if the interstimulus intervals were set to $10 \mathrm{~s}$ or more. As the SBR was not elicited well if the stimulus intervals were shorter than $10 \mathrm{~s}$, we did not study habituation quantitatively by measuring the recovery cycle of paired stimuli. In a patient with early onset Parkinson's disease, a pronounced and stable SBR was present constantly; the highest frequency of stimulation possible to elicit the SBR was 0.5 $\mathrm{Hz}$ (fig 2). In the SBR negative patients, equivocal or unreproducible EMG responses were occasionally obtained; however, these responses did not fulfill the criteria of a positive SBR for the present study.

\section{APPEARANCE OF THE SOMATOSENSORY EVOKED} BLINK RESPONSE

A total of 139 patients with various neurological disorders were examined (table 2) and SBRs were evoked in 23 patients. The mean (SD) latency of the SBRs was $48.5(5 \cdot 7) \mathrm{ms}$ for ipsilateral median nerve stimulation and $52.0(4 \cdot 1) \mathrm{ms}$ for contralateral median nerve stimulation. The SBRs usually showed an ipsilateral predominance. The mean (SD) amplitude of the SBRs was $238.3(160.9) \mu \mathrm{V}$ for ipsilateral median nerve stimulation and $136 \cdot 4$ (113.2) $\mu \mathrm{V}$ for contralateral median nerve stimulation. The mean (SD) duration of EMG activities responsible for the SBR was $45 \cdot 2$ $(14 \cdot 2) \mathrm{ms}$ for ipsilateral median nerve stimulation and $38 \cdot 5(12 \cdot 1) \mathrm{ms}$ for contralateral median nerve stimulation. The appearance of the EMG activities responsible for the SBR varied between stimuli. The lowest intensity stimulus which could evoke the SBR was about twofold the perception threshold. The SBR could occasionally be elicited by stimulation applied not only to the cutaneous surface just above the median nerve trunk at the wrist

Figure 1 Representative $S B R$ s elicited by electrical stimulation of the median nerve (upper) and of the sural nerve (lower). Traces are superimposed.
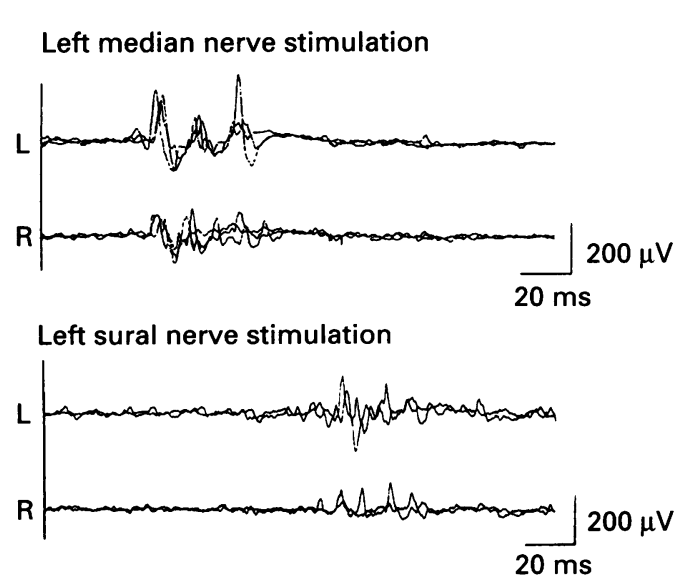

THE STARTLE BLINK AND THE SOMATOSENSORY EVOKED BLINK RESPONSE

In 14 patients (three with sporadic startle disease, two with sporadic olivopontocerebellar atrophy, one with hereditary olivopontocerebellar atrophy, one with thalamic stroke, one with thalamic glioma, one with Meige's syndrome, two with hemifacial spasm, one with early onset Parkinson's disease, one with Guillain-Barré syndrome, one with cerebral infarction), an exaggerated startle reaction was elicited by electrical stimulation of the median nerve. The onset of EMG discharges of the orbicularis oculi muscles responsible for the startle blinks was unclear, and the amplitude of the EMG activities increased gradually and persisted tonically. The latency of the startle blink was always more than $90 \mathrm{~ms}$, which was obviously longer than those of the SBR (fig 3). On the other hand, the EMG burst responsible for the SBR was not tonic but phasic. In four of the 14 patients (one with sporadic olivopontocerebellar atrophy, one with early onset Parkinson's disease, one with hemifacial spasm, one with Guillain-Barré syndrome), the SBR and the exaggerated startle reflex

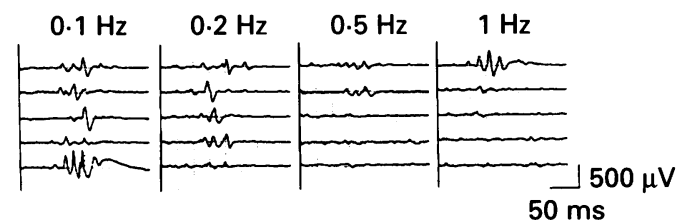

Figure 2 EMGs of the SBR elicited by the repetitive stimulation of the median nerve in a patient with juvenile parkinsonism. This 38 year old woman developed rigidity, akinesia, and disturbance of postural reflexes from the age of 35. All her symptoms responded well to levodopa treatment. 
Figure 3 EMG responses of the startle reaction elicited by left median nerve stimulation in a patient with sporadic startle disease. The EMGs were recorded from the left orbicularis oculi muscle, the left thenar muscle, and the left quadriceps muscle. Two traces are superimposed.
Orbicularis oculi

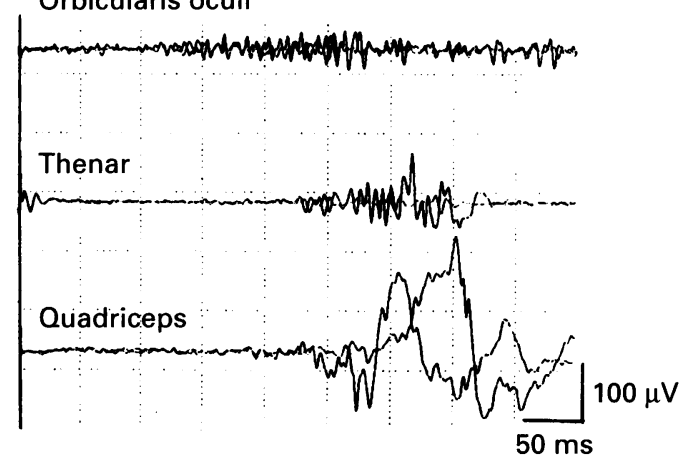

coexisted (fig 4A). The EMG discharges were found to be divided into two responses: the shorter response was identical to the latency of the SBR, and the longer one was identical to the startle blink. The SBR and the startle blink could be elicited either simultaneously or separately.
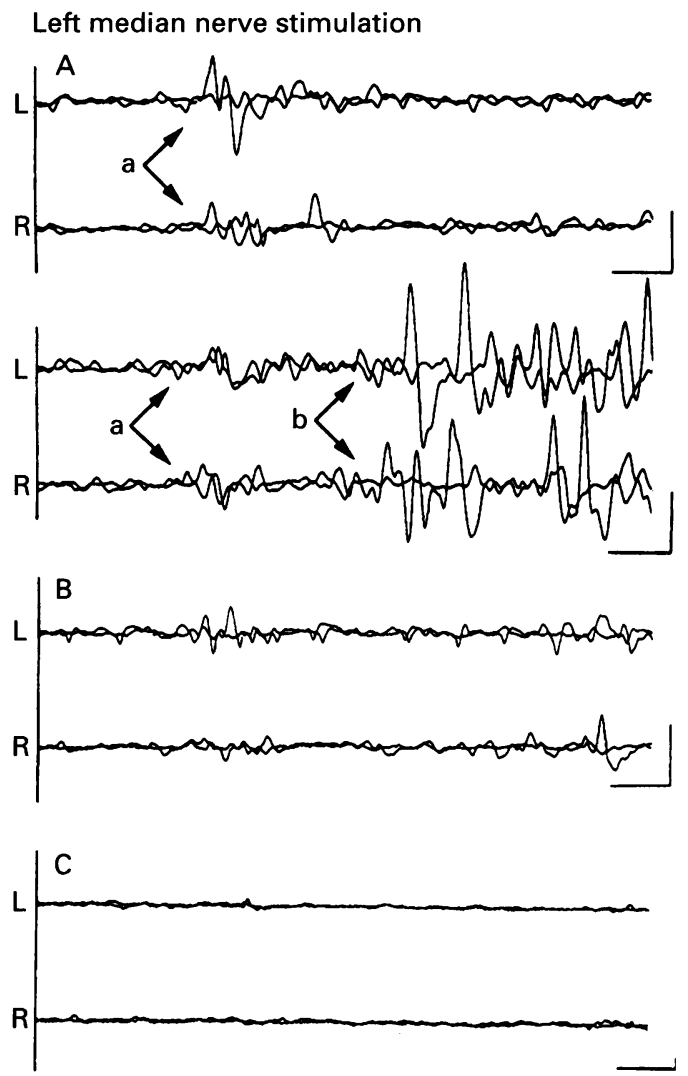

Figure 4 EMGs of the orbicularis oculi muscles after electrical stimulation of the left median nerve in a patient with Guillain-Barré syndrome. This patient was a 40 year old woman who presented with acutely progressive weakness of both upper and lower limbs after a prodoromal infection. The CSF study showed an increase in the protein content with normal cell count. She was diagnosed as having the Guillain-Barré syndrome, and plasmapheresis was performed. After plasmapheresis, her deficits gradually resolved. (A) EMGs at the time of her admission. Arrows $a$ and $b$ indicate EMG activities responsible for the $S B R$ and the startle blink respectively. Note that the SBR appears either with or without the startle blink. Two traces are superimposed. Horizontal and vertical bars indicate 20 $m s$ and $200 \mu \mathrm{V}$ respectively. (B) One week after admission. There is a disappearance of the startle blink. Horizontal and vertical bars indicate $20 \mathrm{~ms}$ and $200 \mu \mathrm{V}$ respectively. (C) Two weeks after admission. The EMG discharge responsible for the SBR became less evident as the patient recovered. Horizontal and vertical bars indicate 20 $m s$ and $200 \mu \mathrm{V}$ respectively.
RELATION BETWEEN THE LOCATION OF LESIONS OR THE TYPE OF DISEASE AND THE

SOMATOSENSORY EVOKED BLINK RESPONSE

Various types of disorders were examined in the present study: cerebrovascular disorders (both infarction or haemorrhage), degenerative disorders (Parkinson's disease, spinocerebellar degeneration), demyelinating disorders (multiple sclerosis), peripheral nerve disorders (Guillain-Barré syndrome, cranial nerve palsy), various forms of dystonia (cranial, segmental, or generalised dystonia), hemifacial spasm, startle disease, and others (brain tumour, Arnold-Chiari malformation, neurosarcoidosis). In the 21 patients with cerebral infarction (both thrombosis and embolism), haemorrhage, brain tumour, Arnold-Chiari malformation or neurosarcoidosis, the lesion site was clearly identifiable by neuroimaging (CT or MRI). The SBR was less elicitable in these patients with a clearly identifiable lesion; in two of the 21 patients, the SBR was positive.

Table 2 shows the presence of the median nerve SBR in various neurological disorders. The SBR was seen most often in patients with both hemifacial spasm or Parkinson's disease. They were less often present in patients with Meige's syndrome, torticollis, or generalised dystonia.

In five SBR positive patients with hemifacial spasm, there was an asymmetric appearance of the SBR (fig 5). In both amplitude and duration, the EMG discharges responsible for the SBR were more pronounced on the side of the hemifacial spasm with either contralateral or ipsilateral limb stimulation. Two of the five SBR positive patients had a familial hemifacial spasm. The two patients were brothers, and they recalled that their father also had facial spasm.

In patients with Parkinson's disease, three of five SBR positive patients had juvenile or early onset parkinsonism with an onset before 40 years old. In one of them, a stereotaxic thalamotomy had been performed, and the SBR disappeared after thalamotomy. There was no relation between the presence of the SBR and that of the poor habituation of the glabella reflex.

Table 2 shows that the SBR was not elicited often in patients with multiple sclerosis or peripheral nerve disorders. One of the two SBR positive patients with a peripheral nerve disorder had Guillain-Barré syndrome. In this patient, the SBR was repeatedly examined, and disappeared during the recovery course of the illness (fig $4, \mathrm{~B}, \mathrm{C}$ ).

\section{BLINK REFLEX STUDIES}

In all the SBR positive patients, the obtained blink reflexes showed normal $R 1$ and $R 2$ responses. Among the SBR negative patients, three patients with an infarction of the lateral medulla, one with brainstem glioma, and one with pontine haemorrhage showed an abnormally depressed appearance of the late response (R2) of the blink. In most of the patients with hemifacial spasm, previously reported findings of the blink reflex were seen, 
Figure 5 Asymmetric SBRs in a patient with hemifacial spasm. This 70 year old woman presented with hemifacial spasm of the left side of her face. Note that the SBR was elicited most prominently on the left side.
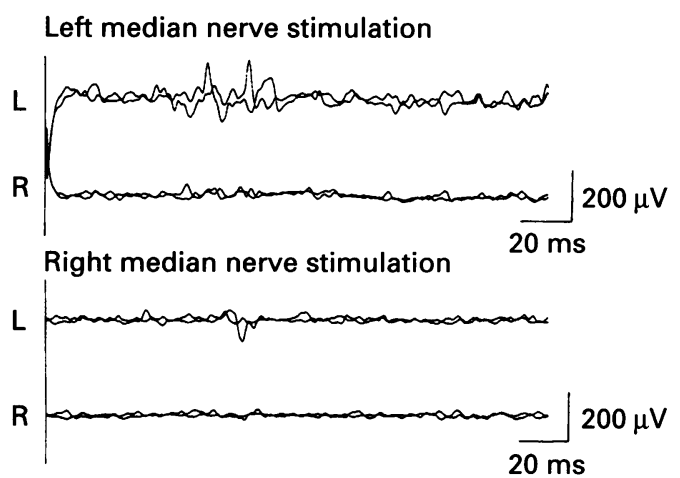

such as prolongation of the $\mathrm{R} 2$ response ipsilateral to the side of the hemifacial spasm, and synkinetic response of the ipsilateral orbicularis oris muscle. ${ }^{89}$

\section{Discussion}

Properties of the SBR found in this study are similar to those reported previously. ${ }^{12}$ The SBR is a reflex phenomenon confined to the orbicularis oculi muscles and is highly susceptible to habituation; its peripheral afferent is possibly of cutaneous origin because the SBR can be evoked by the electrical stimulation of the cutaneous surface of the limbs or of the sural nerve. The present study showed that the SBR can be elicited in some patients with neurological disorders as well as in the normal controls previously reported. ${ }^{1}$ However, the SBR was most often recorded in patients with Parkinson's disease or hemifacial spasm; both of which are known to have increased excitability of the blinking pathway. ${ }^{34}$

SOMATOSENSORY EVOKED BLINK RESPONSE IN NEUROLOGICAL DISORDERS

Hemifacial spasm is electrophysiologically characterised by abnormalities of the blink reflex, such as the presence of the synkinetic response of the ipsilateral orbicularis oris muscle or the presence of delayed or prolonged activities of the ipsilateral orbicularis oculi muscle. ${ }^{8}$ In hemifacial spasm, it has been suggested that a vascular compression of the facial nerve increases excitability of the facial nerve nucleus or the adjacent brainstem neurons. ${ }^{8-10}$ In our study, the SBR was elicited most in the hemifacial spasm on the affected side. Our interpretation of the asymmetric appearance of the SBR in patients with hemifacial spasm is that increased excitabilities within or near the facial nerve nucleus may amplify the magnitude of the outflow required to generate the SBR. Furthermore, the SBR was also elicited in patients with familial hemifacial spasm. Familial hemifacial spasm has been reported rarely. ${ }^{11}$ It remains uncertain whether or not familial hemifacial spasm shares a common pathophysiology with sporadic hemifacial spasm; however, our findings suggest that an increased brainstem excitability may also exist in familial hemifacial spasm.

In Parkinson's disease, increased excitability of neurons in the blink reflex pathway has also been described; some of these patients have poor habituation of the blinking response or a reduced habituation of the blink reflex recovery cycle by paired stimuli. ${ }^{3}$ The presence of the SBR in patients with Parkinson's disease may be related to an abnormal gating mechanism of the sensory-motor linkage within the brainstem reticular formation. ${ }^{12} 13$ On the other hand, in patients with Meige's syndrome or in other patients with dystonia, although dishabituation of the blink reflex recovery cycle has been reported, ${ }^{5-7}$ the SBR was not easily elicitable. The relevance of this difference remains unknown.

In peripheral nerve disorders, the SBR was often absent. However, it is noteworthy that the SBR could only be elicited in a patient with Guillain-Barré syndrome in the acute phase of the illness. We have reported similar findings during the recovery course of patients with Miller Fisher syndrome. ${ }^{1}$ These findings suggest that CNS involvement may exist in Guillain-Barré syndrome. ${ }^{14}{ }^{15}$ Although this is controversial, the presence of the SBR in patients with Guillain-Barré syndrome may indicate that neurons in the brainstem can be stimulated or disinhibited during the acute phase of the Guillain-Barre syndrome.

Multiple sclerosis is characterised by multiple demyelinating plaques in both supratentorial or infratentorial structures. The blink reflex can be used for detecting multiple sclerosis plaques in the brainstem. ${ }^{16-18}$ However, the pathological significance of the presence or absence of the SBR should be considered carefully, because the SBR may appear or disappear with lesions which can facilitate or inhibit the pathways mediating the SBR.

PATHOPHYSIOLOGICAL CONSIDERATION OF THE SOMATOSENSORY EVOKED BLINK RESPONSE

The findings that the pattern of the EMG burst differs between the SBR and the startle blink were compatible with those reported earlier in normal subjects. ${ }^{1}$ These results suggest that the neural pathways mediating the SBR are different from those of the startle reflex. We hypothesise that the SBR is not a specific form of the startle blink, but a variant of blink reflexes. The blink reflex can be evoked by multiple modal sensory stimulations ${ }^{19} 20$ and is a basically programmed response for protecting the eyes from invasive stimuli. Therefore, the SBR may represent a release phenomenon transmitted via the brainstem reticular formation as are the other blink reflexes. ${ }^{21}{ }^{22}$ It may occur as a result of increased excitatory input to the facial nerve nucleus through the reticular formation.

It is also noteworthy that equivocal or minimal EMG responses were occasionally obtained in a subset of the SBR negative patients. These findings were consistent with prior results in normal subjects ${ }^{1}$ and suggest that the SBR is present latently and is habituated under normal conditions. In the continuum to this study, we plan to investigate whether peripheral stimulation has a latent input to the facial nerve nucleus.

Finally, although the SBR is a novel response, the present study shows the possible usefulness of the SBR in hemifacial spasm, 
Parkinson's disease, and some forms of Guillain-Barré syndrome. Future studies will consider the diagnostic and prognostic relevance of the SBR in these diseases.

1 Miwa H, Imamura N, Kogahara K, Ohori T, Mizuno Y. The somatosensory evoked blink response: findings in jects. F Neurol Neurosurg Psychiatry 1995;58:95-9.

2 Imamura N, Miwa H, Hironishi M, Goto K, Mizuno Y. A characteristic blink response observed in a patient with Lance Adams syndrome. Somatosensory-evoked blink rance Adams syndrome. Srain and Nerve (fapan) 1995;47:581-4.

3 Kimura J. Disorders of interneurons in parkinsonism. The orbicularis oculi reflex to paired stimuli. Brain 1973; orbicularis

4 Valls-Sole J, Tolosa ES. Blink reflex excitability cycle in hemifacial spasm. Neurology 1989;39:1061-6.

5 Carella F, Ciano C, Musicco M, Scaioli V. Exteroceptive reflexes in dystonia: a study of the recovery cycle of the R2 component of the blink reflex and of the exteroceptive suppression of the contracting sternocleidomastiod muscle in blepharospasm and torticollis. Mov Disord 1994;9: 183-7.

6 Berardelli A, Rothwell JC, Day BL, Marsden CD. Pathophysiology of blephalospasm and oromandibular dystonia. Brain 1985;108:593-608.

7 Pauletti G, Berardelli A, Cruccu G, Agostino R, Manfredi $M$. Blink reflex and the masseter inhibitory reflex in M. Blink reflex and the masseter inhibitory reflex
patients with dystonia. Mov Disord 1993;8:495-500.

patients with dystonia. Mov Disord 1993;8:495-500.
8 Nielsen VK. Pathophysiological aspects of hemifacial spasm, part 1 (evidence of ectopic excitation and ephaptic spasm, part 1 (evidence of ectopic excitation

9 Nielsen VK. Pathophysiological aspects of hemifacial spasm, part 2 (lateral spread of the supraorbital nerve reflex). Neurology 1984;34:427-31.

10 Møller AR and Jannetta PJ. Blink reflex in patients with hemifacial spasm. Observations during microvascular decompressions. $\mathscr{f}$ Neurol Sci 1986;72:171-82.

11 Micheli F, Scorticati MC, Gatto E, Cersosimo G, Adi J. Familial hemifacial spasm. Mov Disord 1994;9:330-2.

12 Schneider JS. Basal ganglia-motor influence: role of sensory gating. In: Schneider JS, Lidsky TI, eds. Basal gangalia and behavior: sensory aspects of motor functioning. Toronto: Hans Huber 1987:103-21.

13 Lidsky TI, Manetto C, Schneider JS. A consideration of sensory factors involved in motor functions of the basal ganglia. Brain Res Rev 1985;9:133-46.

14 Ropper AH. The CNS in Guillain-Barré syndrome. Arch Neurol 1983;40:397-8.

15 Nadkarini N, Lisak RP. Guillain-Barré syndrome with bilateral optic neuritis and central white matter disease. Neurology 1983;43:842-3.

16 Kimura J. Alterations of the orbicularis oculi reflex by pontine lesions. Study in multiple sclerosis. Arch Neurol 1970;22:156-61.

17 Kimura J. Electrically elicited blink reflex in diagnosis of multiple sclerosis - review of 260 patients over a sevenyear period. Brain 1976;98:413-26.

18 Khoshbin S, Hallett M. Mulimodality evoked potentials and blink reflex in multiple sclerosis. Neurology 1981; 31:138-44.

19 Rushworth G. Observation on blink reflexes. I Neurol Neurosurg Psychiatry 1962;25:93-108.

20 Yates SK, Brown WF. Light-stimulus-evoked blink reflex: methods, normal values, relation to other blink reflexes, and observation in multiple sclerosis. Neurology 1981;31: and observa

21 Holstege G, Tan J, Van Ham JJ, Graveland GA. Anatomical observations on the afferent projections to the retractor bulbi motoneuronal cell group and other pathways possible related to the blink reflex in the cat. Brain Res 1986;374:321-34

22 Hori A, Yasuhara A, Naito $H$, Yasuhara M. Blink reflex elicited by auditory stimulation in the rabbit. $\mathcal{F}$ Neurol $\mathrm{Sci}$ 1986;76:49-59. 